\title{
Pathologisch-anatomische Kasuistik.
}

\author{
Von \\ Prof. Dr. Friedrich Sehlagenhaufer. \\ (Hierzu Tafel I und 2 Textfiguren.)
}

Ich glaube die nachstehenden Fälle in kasuistischer Form an dieser Stelle zur Publikation bringen zu dürfen, um die gynäkologischen Fachkreise auf diese Erkrankungsformen aufmerksam zu machen, zumal über eine Beteiligung der weiblichen Genitalien am chloromatösen bzw. granulomatösen Prozess, soweit ich wenigstens die Literatur überblicke, fast nichts, am lymphosarkomatösen nur wenig bekannt ist.

\section{Ein Fall von Chloro-Lenkämie mit grünem Uterus.}

Ohne hier auf die grossen Kontroversen in der Leukämiefrage eingehen zu wollen, möchte ich einleitend nur bemerken, dass der derzeitige Stand unserer Erkenntnis über das Chlorom etwa dahin geht, dasselbe nur als eine grüngefärbte Variante der gewöhnlichen Leukämie zu betrachten, wobei man unter Leukämie eine maligne Wucherung des hämatopoetischen Systems versteht, die hyperplastisch oder sarkoid verlaufen kann.

Die Grünfärbung wird nur als zufälliges Symptom betrachtet, als Parenchymfarbe aufgefasst. Jede Art der Leukämie, Iymphatische und myeloide, akute und chronische kann einmal als Chlorom verlaufen und zwar sowohl in der hyperplastischen wie in der sarcoiden Form.

Nach dieser kurzen beiläufig orientierenden Einleitung lasse ich meinen Fall von Chloro-Leukämie mit grünem Uterus folgen.

Aus der Krankengeschichte wäre hervorzuheben:

Pat., 26. jähr. Frau, aufgenommen am 22. II.10. Seit 14 Tagen Husten, starke Nachtschweisse. Schwächegefühl, Abmagerung und Blasswerden.

Status praesens: Rötung und Schwellung im Rachen.

Leberschwellung; sie reicht rechts bis zum Darmbeinkamm, in der Mitte bis zum Nabel.

Milz vergrössert unter dem Rippenbogen palpabel. 
Blutbefund: Erythrocyten 1500000 , Leukocyten 60000 .

4. III. Blutungen an den Extremitäten und am Zahnfleisch. Blutung: aus dem Uterus.

5. III, Hochgradige Schwäche. Temp. 38,8.

8. III. Schwellung der Drüsen anf der linken Gesichtsseite. Temperatur 39.2. Abends Exitus.

Klinische Diagnose: Leucaemia acuta. Angina tonsillaris.

Die am 9. März ausgeführte Obduktion ergibt auszugsweise folgenden Befund: Hautblutungen. Grünlich gefärbte Auflagerungen an den Schädelknochen and auf der Dura. Schmutzig grüngefärbte knotige Infiltrate der grossen Sichel. Im Plexus choroideus 2 grünliche Knoten. Bis nussgrosse grüne Knoten im Unterhautzellgewebe der Brust, zwischen den Brustmuskeln und im Mammagewebe. Grünfärbung der Knorpelansätze der Rippen.

Flächenhafte und knotige, grasgrüne, dicke Infiltrate an beiden Seiten des Sternums. Die vergrösserten Halslymphdriisen ohne Grünfärbung.

Beide Tonsillen vergrössert, schmutzig grün; beim Einschneiden entleert sich übelriechender, fetziger, grünlicher Inhalt. Follikel am Zungengrund vergrössert, schmutzig grau.

Grüne Knoten und grüne flächenhafte Infiltrate an der Herzmuskulatur und am Epikard und in der Gefässscheide der grossen Gefässe. Grüngefärbte bronchiale Drüsen. Zahlreiche grüngefärbte Knoten und Infiltrate im parietalen Blatt des Peritoneum, im grossen Netz, im Mesokolon und den Appendices epiploicae.

Milz $480 \mathrm{~g}$ schwer, $20 \times 12 \times 6 \mathrm{~cm}$. Einige graugrüne Herde an der Oberfläche. Am Durchschnitt die Pulpa graurötlich mit einer grünlichen Nuance. Grüngefärbte Stellen sieht man nur an der Peripherie der grösseren Gefässdurchschnitte. Hilusdrüsen der Milz vergrössert, grïn.

Leber $3030 \mathrm{~g}$ schwer; plump; diffus infiltriert.

In beiden Nieren prächtig grüne arkadenartig zwischen Rinde und Mark sich ausbreitende Infiltrate und grine Knoten.

Im Douglas, in der Excavatio vesicouterina und rectouterina, im peritonealen Ueberzug des Uterus und auf den Ovarien bald flache, bald buckelige grüne Infiltrate. An der hinteren Fläche des Corpus uteri wölbt sich ein griuner Knoten über das Niveau hervor.

Der etwa $10 \mathrm{~cm}$ lange Uterus ist fast diffus graugrün gefärbt. Nach Eröfuung desselben und Ausräumnng eines dunkelroten Blutgerinnsels erscheint die Schleimhaut des Fundus wie des Cervicalkanals durch ein grasgrünes Infiltrat verdickt. Reichliche Blutpunkte im Carum, im grünen Infiltrat geben ein buntes Farbenbild. Auch die Muskelschichten, die im Corpus etwa $2 \mathrm{~cm}$, im Cervix $1 \mathrm{~cm}$ dick sind, sind durch eine grüne Aftermasse infiltriert, die zum Teil diffus die Uteruswand durchsetzt, zum Teil in Form grüner Knoten eingesprengt ist. Die vordere und hintere Muttermundslippe ist durch grüne knotige Infiltrate wulstig verdickt. In der Vagina normale Verhältnisse. Taf. I Fig. 1.

Sonst fanden sich noch reichliche grüne Periostinfiltrate zu beiden Seiten der Wirbelsäule und an der Vorderseite des Kreuzbeines. 
Die histologische Untersuchung ergibt: Sämtliche Schichten der Gebärmutter sind von einem mehr weniger dichten Infiltrate gleichförmiger Zellen durchsetzt.

In den Muskelschichten ist. hauptsächlich das Bindegewebe dicht infiltriert. Aber auch die Muskelbündel selbst sind oft durch die infiltrierenden Zellen auseinander gedrängt.

Die Schleimhaut des Uterus ist deutlich zu sehen, aber meist so dicht infiltriert, dass die Drüsen des Fundus nur mehr in den tieferen Schichten vorhanden sind. Dagegen sind die Cervixdrüsen in normaler Menge da, nur von einem dichten Wall der Infiltrationszellen umgeben. Auch in den Ovarien und in den Parametrien sind oft dichte Haufen derselben Zellinfiltration zu sehen. -

Die Zellen, aus welchen die Infiltrate sich zusammensetzen, entsprechen den im Blute vorhandenen grossen mononuklearen Leukocyten.

Im Triacidschnittpräparat färbt sich der grosse lockere Kern schwach blau, das Protoplasma wechselnd stark rötlich. Nach Giemsa färbt sich Kern und Protoplasma blau, letzteres sehr schwach.

Die Winkler-Schultze'sche Oxydasereaktion ist negativ.

Der vorstehende Fall ist demnach ein akut verlaufendes Chlorom bzw. eine Chlor-Leukämie, genaver rubriciert eine LymphoblastenChlor-Leukämie.

Was ihn besonders auszeichnet und weswegen er überbaupt publiciert wurde, ist die durch die leukämische Infiltration der Uteruswände und -schleimhaut erzeugte Grünfärbung der Gebärmutter. Wir können von einem graugrünen Uterus sprechen.

Diese auch an der Portio vaginalis deutlich sichtbare Grünfärbung könnte gelegentlich ähnlich wie grüngefärbte Haut und Schleimhautinfiltrate oder grüngefärbte Follikel am Zungengrund zur Diagnose eines chloromatösen Prozesses führen, dessen Differentialdiagnose gegenüber anderen ILeukämien ja immer noch klinisch auf grosse Schwierigkeiten stösst.

Freilich scheint die Beteiligung des Uterus am chloromatösen Prozess eine sehr seltene zu sein, denn die Literatur erwähnt nichts davon.

\section{Ein Fall von Lymphosarkom des Uterus und der Adnexe.}

Die Zahl der publicierten Lymphosarkome des Uterus ist eine sehr geringe. Und die meisten der Fälle halten einer kritischen 
Prüfung nicht stand. So sind die 3 von Gessner im Handbuch der Gynäkologie angeführten Lymphosarkome von Wilischanin, Gow und Jassorin kaum als solche anzuerkennen.

Gow und Jassorin geben eine ganz kurze Schilderung ohne eine histologische Untersuchung and Wilischanin betrachtet wohl auf Grund seiner histologischen Untersuchung seine Uterusgeschwulst als ein Lymphosarkom. Aber die Beschreibung des Tumors als eine pilzartig in die Höhle der $7 \mathrm{~cm}$ langen Gebärmutter hineinragende Wucherung, die die rechte Seite des Uterus einnimmt und sich in die Richtung des Eileiters verbreitert und an die Oberfläche tritt, ist wenig geeignet, die Richtigkeit der Diagnose zu stützen.

Auch der von R. Lerchenthal in einer Inaugural-Dissertation "Ueber Uterussarkome" geschilderte Fall eines Lymphosarkoms des Uterus, lassen Zweifel aufkommen, ob wir es mit einem Lymphosarkom im Sinne Kundrats zu tun haben, oder vielmehr um eine Beteiligung des Uterus bei einer Leukämie.

Er beschreibt sein Präparat folgendermassen: „Gebärmutter $6 \mathrm{~cm}$ lang, Cervix $2 \mathrm{~cm}$, Wanddicke 2,3 cm. Die Cervix setzt sich ziemlich scharf vom Körper ab. Während die Muskelwand stets von annähernd gleicher Stärke bleibt, bildet die Schleimhaut einen deutlich hervorspringenden Wulst. Dieser an seiner dicksten Stelle $1,3 \mathrm{~cm}$ betragend, ist gegen die Muskelwand gut abgegrenzt. Tuben beiderseits stark verdickt, die linke etwa doppelt so stark wie die rechte.

Diese Beschreibung stimmt nicht mit dem überein, was wir sonst bei einem Lymphosarkom anderer Organe, z. B. des Magens, des Cöcum, des Rectum zu sehen gewöhnt sind. Der Uterus ist klein, wenig verdickt.

Jedenfalls entspricht das Präparat nicht dem Bilde, das uns Wagner von seinem Lymphosarkom gibt, noch weniger unserer eigenen Beobachtung.

Wagner schildert sein unzweifelhaftes Lymphosarkom wie folgt: Cervix und unteres Uterussegment ist von einer weissen, etwas faserigen Neubildungsmasse infiltriert, so dass die Wand des $8 \mathrm{~cm}$ langen Cervicalkanals $4 \frac{1}{2} \mathrm{~cm}$ dick ist.

Die Vaginalportion ist an der vorderen Lippe wie anch seitlich $4,5 \mathrm{~cm}$ breit und geht in die von derselben Aftermasse infiltrierte und verdickte vordere Vaginalwand über.

Dieser förmlich cylindrischen Geschwulst sitzt das dünnwandige, 
erweiterte Corpus auf, in dessen Wandschichten sich ebenfalls Infiltrationen der weissen Aftermassen finden.

Parametrien und beide Tuben sind infiltriert und stellen glatte, dicke Wülste vor.

Beide Lig. rotunda sind von der Neubildung ergriffen, das linke in Form eines fingerdicken Zapfens, das rechte in Form mehrerer konfiaierter Knoten.

Da auch die histologische Untersuchung - Zellen von lymphatischem Charakter in einer retikulären Grundsubstanz - den Befund eines Lymphosarkoms ergibt, so erklärt Wagner mit Recht, dass die Geschwulst makro- und mikroskopisch vollkommen dem Typus der Lymphosarkome entspricht.

Meine eigene Beobachtung ergibt einen sehr ähnlichen vielleicht noch typischeren Befund.

Das Präparat stammt von einer 40 jährigen Frau mit starken Uterusblutungen. Der Status gynaecol. lautet $1^{1 / 2}$ Monate vor dem Tode: Uterus über kindskopfgross, derb, in seiner Beweglichkeit beschränkt. Beiderseits faustgrosse fixierte Adnexresistenzen. Cervicalkanal ofien; die Portio roluminös aufgetrieben, die Sonde stösst stellenweise an.

Die am 21. Oktober 1907 vorgenommene Obduktion ergab: Der Uterus ist in allen seinen Dimensionen stark vergrössert und mit der Umgebung fixiert.

Seine Länge vom Fundus bis zum Orificium externum beträgt $17 \mathrm{~cm}$. Seine grösste Breite ist $10 \mathrm{~cm}$, die grösste Dicke $6 \mathrm{~cm}$. Seine Konfiguration ist dabei gut erhalten und der cervicale Anteil deutlich rom Corpus abgrenzbar. Die voluminöse Portio vaginalis klafft und hat einen Durchmesser von $5 \mathrm{~cm}$. Diese starke Vergrösserung des ganzen Uterus ist bedingt durch eine diffuse, nur am Corpus buckelig sich vorwölbende weissgraue Aftermasse.

Am eröffneten Uterus sieht man, wie die Muskelwand und die Schleimhaut im Fundos wie im Cervix durch ein weisslich graues Geschwulstgewebe infiltriert sind.

Ganz monströs sind die vordere und hintere Muttermundslippe vergrössert. Die Cervicalportion ist ca. $10 \mathrm{~cm}$ lang, die Wanddicke $2 \mathrm{~cm}$ und darüber.

Das Corpus uteri misst $7 \mathrm{~cm}$, seine Wanddicke über $3 \mathrm{~cm}$. Die Uteruswände sind durch die infiltrierenden Tumormassen derb und starr. Beide Tuben sind in den Geschwulstmassen der Adnexe gut differenzierbar. Die rechte Tube ist am uterinen Ende federkieldick, im Isthmus verjüngt sie sich, schwillt dann wieder an und bildet am abdominalen Ostium einen recht grossen Geschwulstknoten. Die linke Tube ist fast durchaus kleinfingerdick, am Durchschnitt erkennt man aber deutlich die gefaltete Tubenschleimhaut; das abdominale Ende geht in eine Geschwulst über.

Beide Orarien sind kleinfaustgross und durch ein weissrötliches Aftergewebe substituiert. Im allgemeinen ist aber doch die Konfiguration der Ovarien zu erkennen und an Durchschnitten sieht man auch stellenweise Follikelcysten und die eigentümlichen Strukturverhältnisse des Eierstockes. Die Parametrien sind diffus infịltriert und dick. 
Aus dem übrigen Sektionsbefund wäre noch hervorzuheben: Knotige Infiltrate in der Magenschleimhaut, diffuse Infiltration des grossen Netzes, der Dünndarmserosa und des Wurmfortsatzes.

Histologisch besteht das Geschwulstgewebe aus gleichförmigen lymphocytären Zellen in einem retikulären Grundgewebe. Alle Gewebsschichten des Uterus sind infiltriert; desgleichen die Ovarien und die Tubenwände, wobei die Schleimhaut selbst oft ganz erhalten ist.

Figur 1 (ca. 1/3 der natürlichen Grösse).

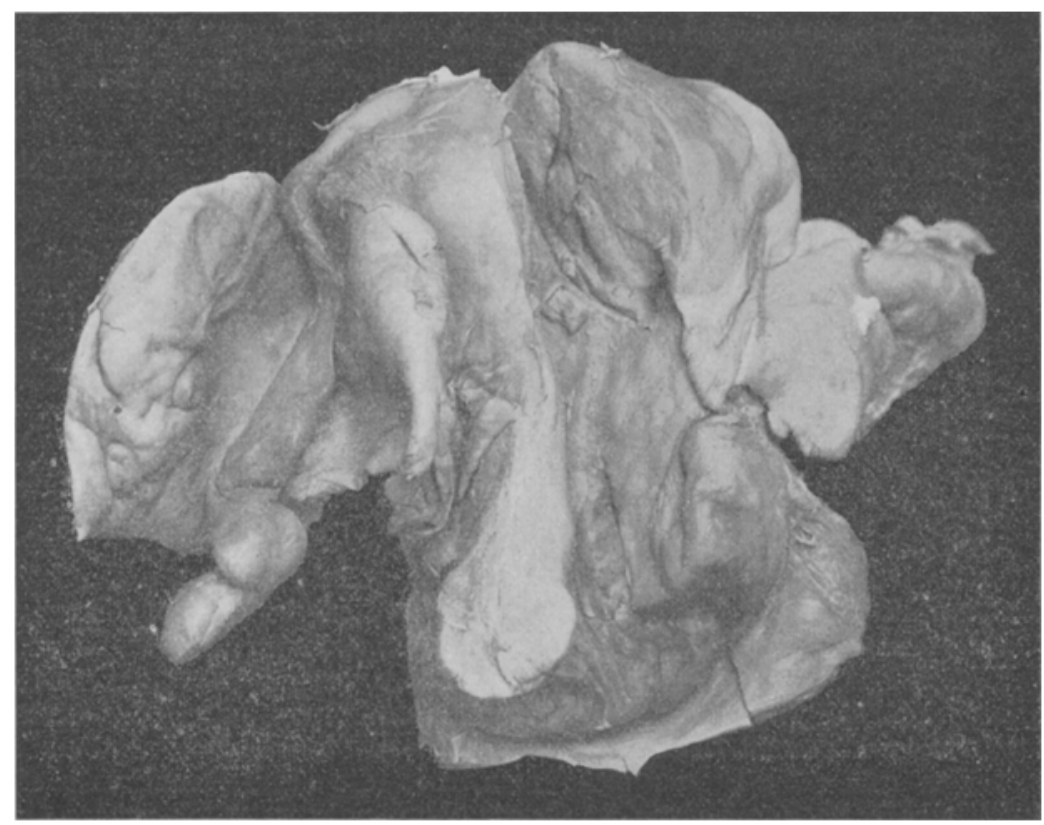

Wir glauben, dass durch diese beiden Fälle, Wagners und unsere eigene Beobachtung, der Typus des Lymphosarkoms des Uterus und der Adnexe gezeichnet ist, und dass nur ein makroskopisch derart sich verhaltendes Präparat als echtes Lymphosarkom im Sinne Kundrats angesehen werden dürfe.

Und zwar möchten wir den makroskopischen Befund fïr entscheidender ansehen als den mikroskopischen, denn es ist nur zu oft unmöglich die histologische Differentialdiagnose zwischen Lymphosarkom und leukämischer Infiltration oder Rundzellensarkom zu stellen.

Als Ausgangspunkt der Neubildung müssten wir präexistierendes adenoides Gewebe (Kundrat) etwa in der Portio vaginalis 
betrachten, wofür die Befunde Lesniowskis und Hausers über gehäuftes Vorkommen echter Lymphfollikel in jener 'Schleimhautregion des Uterus eine Stütze bilden würden.

\section{Zwei Fälle von Grannlomatosis (Typus Paltanf-Sternberg) mit Beteiligung der Adnexe.}

Es scheint auch hier angezeigt, einleitend eine beiläufige Skizze des derzeitigen Standes unserer Kenntnisse über die Lymphogranulomatosis (Typus Paltauf-Sternberg) zu geben.

Diese eigentümliche Form der Erkrankung des lymphatischen Systems beginnt gewöhnlich an irgend einer Gruppe von Drüsen, meist am Halse und führt zu einer derben Vergrösserung der Lymphdrüsen, die oft zu mächtigen Paketen anschwellen. Unter Fieberattacken, die meist intermittierenden Typus zeigen, verallgemeinert sich in der Regel der Prozess.

Es kommt zu knotigen Einlagerungen in der Milz (Porphyrmilz); der Leber, im Knochenmark, in den Iungen,. seltener in anderen Organen.

Klinisch wird die Diagnose gestützt durch den Blutbefund einer absoluten neutrophilen polymorphkernigen Lueukocytose mittleren Grades, eine positive Diazoreaktion und fortschreitende Kachexie.

Das Charakteristische aber ist der histologische Befund. Es kommt im lymphatischen System zur Bildung eines eigenartigen Granulationsgewebes, das sich durch seinen oft sehr bedeutenden Reichtum an überaus grossen Zellen auszeichnet, deren Protoplasma reichlich, deren ein- oder mehrfache Kerne sich meist intensiv färben. Dazu gesellen sich epithelioide Zellen, neutrophile polymorphkernige Leukocyten, Eosinophile, Plasma- und Mastzellen in wechselnder Menge.

Im weiteren Verlauf treten Herde vom Charakter hyaliner Nekrosen auf; die Fibroblasten werden immer zahlreicher, es kommt zur reichlichen Bindegewebsentwicklung, oft zu einer Fibrosis. In der Regel bleibt der Prozess auf das lymphatische System beschränkt.

In anderen Fällen zeigt aber das Granulationsgewebe aggressiven Charakter. Es greift auf die Umgebung über, infiltriert Perikard, Pleura, Lungen usw.; bricht in die Venen ein. Es trägt geschwulstartigen Charakter. Diese sarkoiden Formen waren auch der Grund, dass manche Autoren den ganzen Prozess als Sarkom betrachtet 
wissen wollen. Ich füge hinzu mit Unrecht. Was die Pathogenese und Aetiologie betrifft, so herrscht noch ziemliches Dunkel.

Sternberg hat seinerzeit derartige Fälle als eigenartige unter dem Bilde der Pseudoleukämie verlaufende Tuberkulose des lymphatischen Apparates beschrieben. Diese Ansicht wurde ebenso entschieden bekämpft.

Derzeit stehen im Vordergrund des Interesses die Befunde Fraenkels und Muchs, die im granulomatösen Gewebe antiforminfeste, nicht nach Ziehl, aber nach der modificierten GramMethode färbbare Granula und granulierte Stäbchen nachweisen konnten, die morphologisch von der granulierten Form des Tuberkelbacillus nicht zu unterscheiden sind.

Gross ist bereits die Literatur über diesen Gegenstand. Sie wurde jünst von Fabian in einem Sammelreferat zusammengefasst, das 461 Arbeiten enthält.

Wie aus denselben hervorgeht und wie ich durch eigene Literaturstudien bestätigen kann, ist bis jetzt eine Beteiligung der weiblichen Geschlechtsorgane an der Granulomatosis fast nicht beschrieben.

Fabian citiert nur Hérard (Cornil), der im Jahre 1865 im Archive générale eine Miterkrankung der Ovarien an Hodgkins disease beschrieben haben soll. Leider war weder Fabian, wie er mir schriftlich mitteilte, noch auch ich selbst in der Lage, die Originalarbeit zu lesen. Wenn wir aber die Zeit ihres Erscheinens berücksichtigen, dürfte sie schwerlich namentlich mikroskopisch verwertbar sein.

Es scheint mir daher gerechtfertigt meine 2 Fälle hier kasuistisch mitzuteilen.

Fall 1. Elisabeth Sch., 50jährige Frau, aufgenommen am 14. X. 1910, gestorben am 7. XII. d. J.

Ich entnehme der Krankengeschichte, die ich Herrn Primarius Doc. Dr. R. Schmidt verdanke, etwa folgendes.

Pat. hat seit einem Jahre Schmerzen in der linken Bauchseite. Seit einem halben Jahre bemerkte sie dort eine Geschwulst, die langsam an Grösse zunahm.

In den letzten Monaten Nachtschweisse. Letzte Menses Mitte August d. J. 3 Partus, 1 Abortus.

Status somaticus. Ueber dem rechten Oberlappen verkürzter Schall, verschärftes Atmen. Herz normal. Abdomen vorgewölbt, weich. 4 Querfinger unter dem linken Rippenbogen eine apfelgrosse, derbe höckerige bewegliche Geschwulst zu tasten. In inguine beiderseits Drüsen zu tasten.

Status gynaecologicus: Tumormassen hinter dem Uterus. Derselbe retroflektiert, nach rechts verdrängt. Der Tumor fühlt sich derb an. 
Decursus: 20. X. Starke Kopfschmerzen; abends Fieber.

29. X. Abends immer Temperatursteigerungen, häufiger Husten.

7. XI. Abends Temperatur bis $38^{\circ}$. Starke Nachtschweisse.

25. XI. Temperatur normal. Nachtschweisse und Husten geringer. Grosse Somnolenz.

2. XII. Temperatur normal.

4. XII. Fortschreitender Verfall.

6. XII. Morgentemperatur $38^{\circ}$. Sensorium getrübt, nachmittags. Schweissausbruch. Temperatur 36,6.

7. XII. Exitus.

Harnbefund: Diazoreaktion stark positiv, Aldehyd positiv Cutanreaktion negativ. Leukocyten 20000 , hauptsächlich polymorphkernige Leukocyten. minis.

Klinische Diagnose: Neoplasma malignum gland. lymph. abdo-

Die am 8. XII. vorgenommene Obduktion ergab folgendes: Mittelgrosse, weibliche Leiche von gracilem Knochenbau. Haut blass, geringer Panniculus adiposus.

Supraclavicular und in inguine beiderseits derbe Drüsen tastbar. Tonsillen, Follikel am Zungengrund nicht vergrössert. Schilddrüse, Kehlkopf, Trachea normal.

Die vergrösserten, derben Drüsen in den Supraclaviculargruben zeigen ein erweichtes Centrum.

Herz, Gefässe normal; ebenso Mediastinum.

Die rechte Lunge ist im Oberlappen fast luftleer, fühlt sich wie pneumonisch an. An der Oberfläche sieht man grauweisse derbe bis zu $0,5 \mathrm{~cm}$ dicke strangförmige Infiltrate an der visceralen Pleura. Am Durchschnitt ist das Lungengewebe von bald breiten, bald schmalen Streifen eines weisslichen Aftergewebes durchzogen, das auch zum Teil die Alveolen erfüllt und an der Lungenwurzel die anthrakotischen Drüsen umscheidet. Im Mittellappen sind 3 derbe, wallnussgrosse, weissliche Knoten. Im Unterlappen sind nur streifige Infiltrate in der Pleura, die an einer Stelle plaqueartig. werden und zu einem haselnussgrossen, grauweissen derben Knoten sich verdichten. Die linke Lunge enthält im Oberlappen einige kleine, graue Knötchen; im Unterlappen sind mehrere hasel- bis wallnussgrosse derbe Knoten.

Die Pleura costalis zeigt auf beiden Seiten kleine und grössere flächenhafte weissliche Infiltrate.

Nach Eröffnung des Abdomens sieht man den Dickdarm bis zur Flexura lienalis stark gebläht. Das Colon descendens ist kontrahiert und durch Drüsenpakete, welche die ganze linke Bauchregion einnehmen, an die Bauchwand gedrückt,. Das über faustgrosse Drüsenpaket besteht oberflächlich aus einzelnen Knollen, die eine ziemlich weiche Konsistenz besitzen und von gelber Farbe sind. Die Knollen lassen sich zum Theil isolieren, zum Theil sind sie aber untereinander verwachsen.

Am Durchschnitt ist die Mitte der einzelnen Knoten meist nekrotisch und auch das Centrum des massigen Drüsentumors ist erweicht und von nekrotischen, gelblichweissen, rahmigen Massen erfüllt.

Die Leber zeigt normale Grösse, Farbe und Struktur. Am Ansatz des Lig. falciforme und triangulare dextrum sind einige knotige grauweisse Infiltrate.

Die Milz ist etwa auf das Doppelte vergrössert, von normaler Konfiguration. Beim Betasten fühlt man deutlich derbere Partien. Am 
Durchschnitt sieht man nur undeutlich Knoten, die sich zum Teil durch ein gelbliches nekrotisches Centrum von der Umgebung abheben. Die Pulpa ist weich, blutreich.

Pankreas, Nebennieren, Nieren, Magen und Dünndarm normal.

Am Colon sind fast sämtliche Appendices epiploicae durch eine Aftermasse infiltriert, so dass sie kirschkerngrosse derbe, weissliche Knoten bilden.

Im kleinen Becken ist ein mit den linken Adnexen in Verbindung stehender mannsfaustgrosser Tumor. Nach Entnahme des Genitales finden sich folgende Verhältnisse: Vagina und Uterus normal. Rechts ist der Isthmus tubae uterinae etwa in einer Ausdehnung von $2 \mathrm{~cm}$ durch eine weissgelbliche Aftermasse infiltriert. Im Mesovarium ist ein erbsengrosser weissgelblicher Knoten.

Auf der linken Seite lassen die höckerigen, mehr weichen, gelbrötlichen Geschwulstmassen keine Differenzierung der einzelnen Adnexbestandteile erkennen. Nur einige cystische Gebilde deuten den Sitz des Ovariums an wad tentakelartige Geschwulstwärchen, die die Gestalt der Fimbrien imitieren, weisen auf das abdominelle Ostium der Tube hin (Taf. I, Fig. 2). Man kann auch daselbst die Tube eine Strecke weit sondieren und beim Aufschneiden erkennt man deutlich die Tubenschleimhaut, die ganz in Aftermassen eingebettet ist.

Granulomherde in der Wirbelsäule und in den langen Röhrenknochen.

Nach der Sektion konnte keine bestimmte Diagnose gestellt werden, doch wurde der Verdacht anf "Granulomatosis Typus Paltauf-Sternberg" ausgesprochen.

Ganz eindeutig war das Ergebnis der histologischen Untersuchung. Drüsen, Lungeninfiltrate und -Knoten, Milz, Knochenherde zeigen alle das belzannte polymorphe Granulationsgewebe mit zahlreichen grossen Zellen mit ein oder mehreren intensiv gefärbten Kernen und wechselnden Mengen Lymphocyten, polymorphkernigen Leukocyten und wenigen Plasmazellen.

Ganz denselben Charakter zeigte auch das geschwulstartig wochernde Gewebe der Adnexe. Ueberaus sehön ist dies am Fimbrienende der Tube zu sehen (s. Fig. 2).

An Stelle der Tubenwand ist ein aus verschiedenen Zellen namentlich aber aus polymorphkernigen grossen, intensiv gefärbten Zellelementen aufgebautes Gewebe getreten, das Muskelwände und Bindegewebe völlig substituiert, dem aber ganz intakt das Epithel der Tubenschleimhaut auflagert.

Auch das Ovarium ist durch jenes eigentümliche Zellgewebe substituiert und nur die Corpora candicantia, die meist ganz yom granulomatösen Gewebe respektiert werden, lassen die Diagnose: Eierstock zu. 
Ebenso zeigen die Infiltrate und Knoten in den Adnexen denselben histologischen Charakter.

Nirgends Zeichen von Tuberkulose. Ein Meerschweinchen mit Geschwulstpartikelchen intraperitoneal geimpft bleibt gesund. Die Suche nach Fraenkel-Much'schen antiforminfesten granulierten Stäbchen blieb erfolglos.

Fall 2. Derselbe betrifft eine 68 jährige Frau, die am 26. Januar 1911 zur Sektion kam. In diesem Fall war der Hauptsitz der Granulomatosis mit sarkoidem Charakter die Milz, das Pankreas und die abdominellen Lymphdrüsen.

Das Genitale war folgendermassen beteiligt: Vereinzelte meist erbsengrosse gelbliche derbe Knoten im Mesosalpinx und im Mesovarium beider Seiten.

Figur 2.

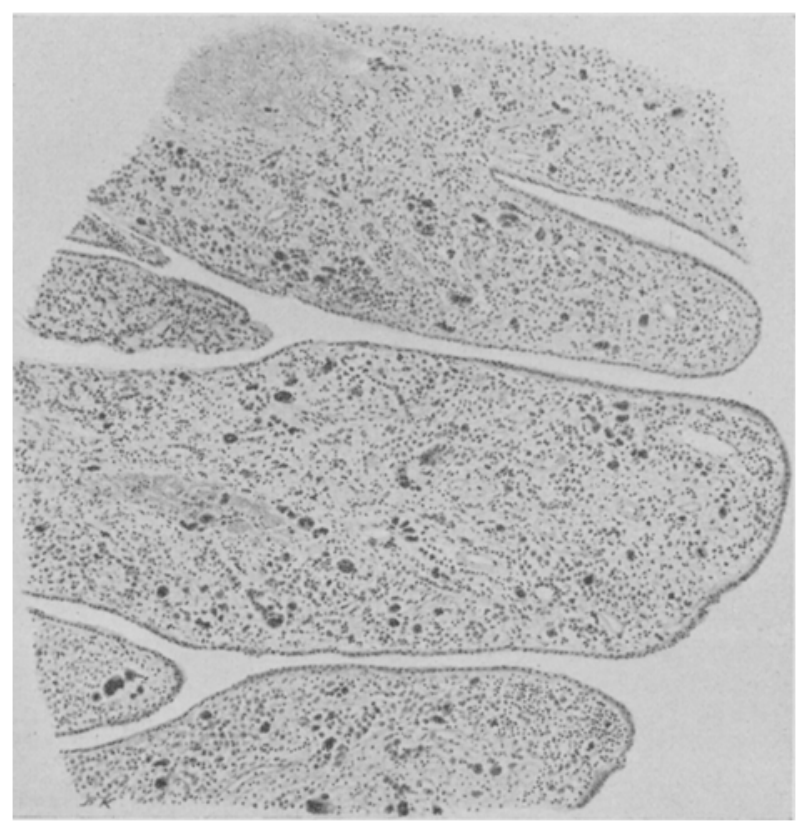

Ovarien-Eileiter, Uterus normal. Sehr zahlreich und fast miteinander konfluierend sind gelbliche Knoten und Infiltrate in beiden Parametrien vorhanden.

Der Plexus utero-vaginalis ist derb infiltriert. Am Durchschnitt scheinen die Aftermassen zum Teil in Venen zu stecken (Taf. I Fig. 3).

Vagina normal. In der Blasenschleimhaut sowie in der Urethra und im periurethralen Bindegewebe mehrere Infiltrate und derbe Knoten.

Histologisch entsprach der ganze Fall wieder völlig der sarkoiden Form der Granulomatosis Typus Paltauf-Sternberg: und auch 
die Knoten und Infiltrate in den Adnexen boten das entsprechende Bild. Hervorzuheben wäre, dass hier an verschiedenen Stellen der Parametrien ein Einbruch des Granuloms in Venen nachgewiesen werden konnte. Fraenkel-Muchsche Bazillen konnten nicht gefunden werden.

\section{Zusatz bei der Korrektur.}

Die praktische Wichtigkeit der Kenntnis der Beteiligung der Geschlechtsorgane am granulomatösen Prozess wird schön illustriert durch den von Luce in der medizinischen Klinik vom Mai 1911 veröffentlichten "klinisch ejgenartigen Fall von Hodgkinscher Krankheit (Typus Paltauf-Sternberg) mit Fraenkel-Muchschen Bazillen-Befund."

Im Verlaufe eines recht dunklen, lange als Paratyphus angesehenen Falles ergab die gynäkologische Untersuchung einen anscheinend faustgrossen linksseitigen Adnextumior. Bei der Laparotomie fand sich zwischen den Platten des Lig. latum sinistr. eingeschlossen ein apfelgrosser Tumor. Es gelang die Ausschälung der Geschwulst. Sie erwies sich histologisch als ein Granulom (Typus Paltauf-Sternberg). Simmonds und Fraenkel konnten im antiformierten Präparate granulierte, gramfärbbare Bazillen ganz vereinzelt auffinden.

Jetzt erst konnte die richtige Diagnose des Falles gemacht werden. Sektion wurde verweigert.

\section{Erklärung der Abbildungen anf Tafel I.}

Fig. 1. Chloromatöser Uterus; fast natürliche Grösse.

Fig. 2. Granulomatosis der linken Adnexe; $1 / 2$ der natürlichen Grösse.

Fig. 3. Granulomknoten in den Parametrien; $2 / 3$ der natärlichen Grösse. 

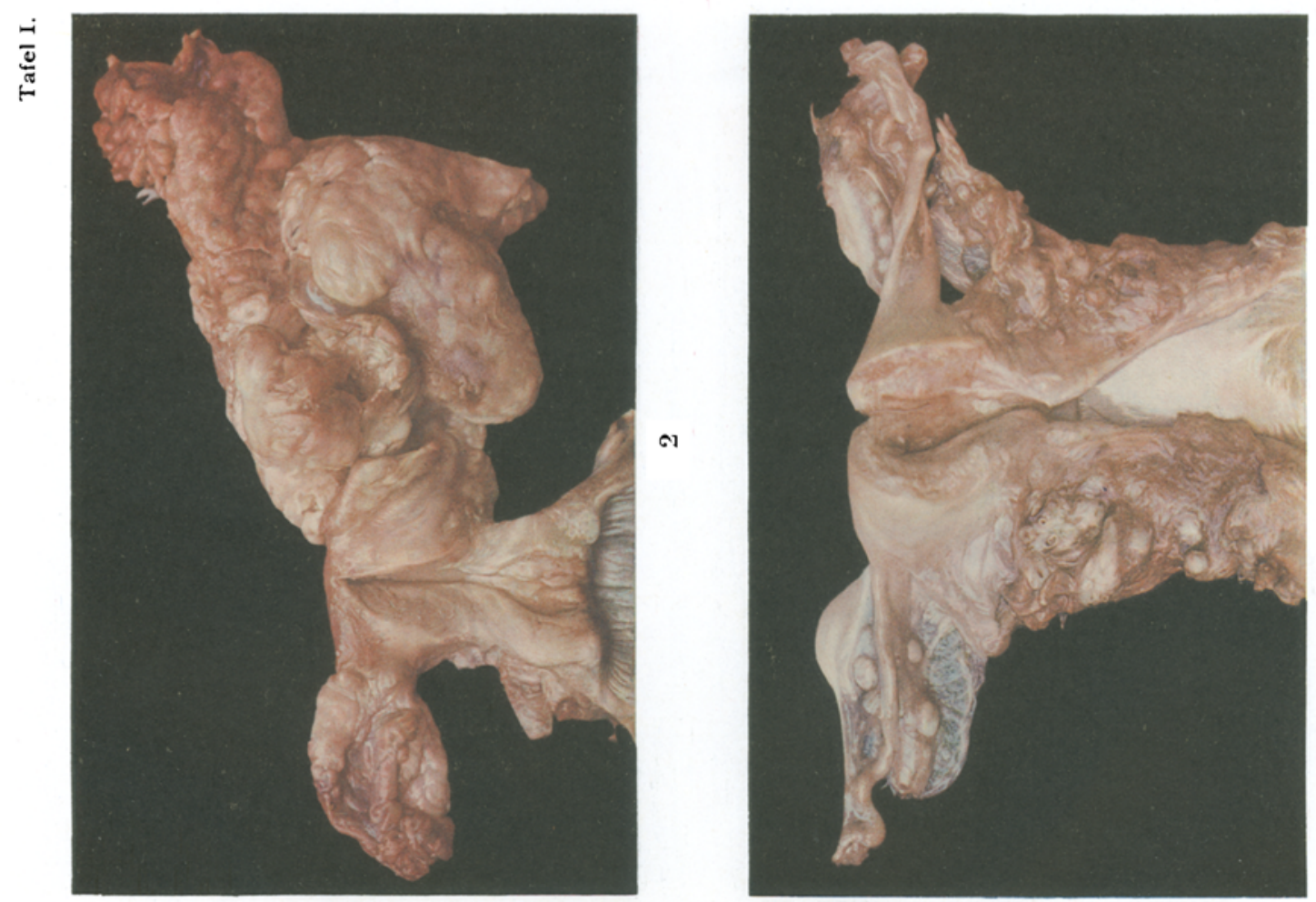

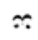

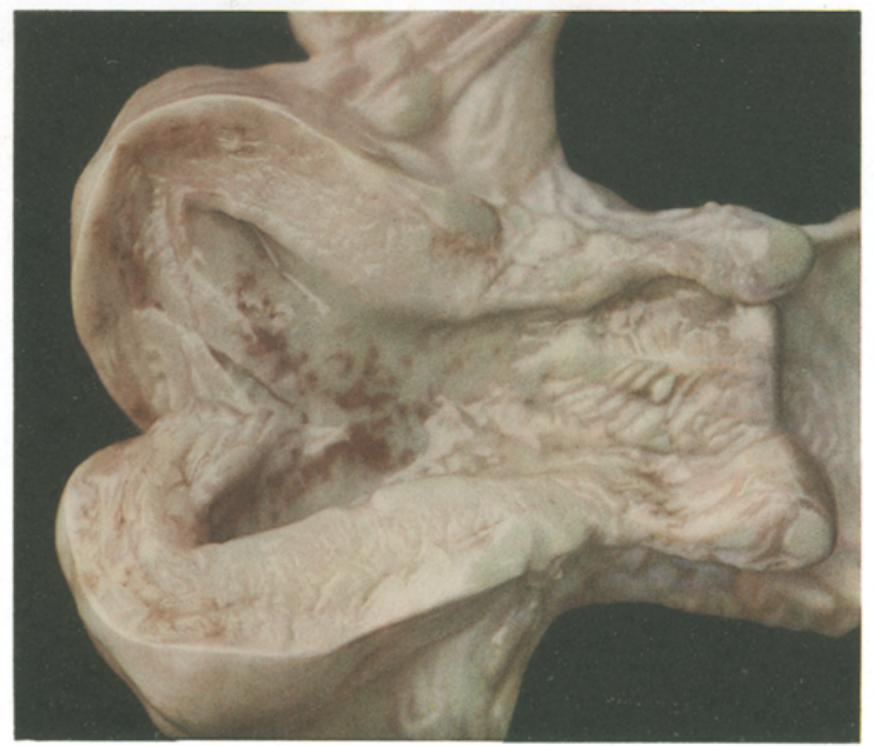

\begin{tabular}{c}
2 \\
\hline
\end{tabular} 